\title{
Expression levels of seprase/FAP $\alpha$ and DPPIV/CD26 in epithelial ovarian carcinoma
}

\author{
MENGZHEN ZHANG, LIWEI XU, XIAOLING WANG, BEIBEI SUN and JUAN DING \\ Department of Obstetrics and Gynecology, The First Affiliated Hospital of Zhengzhou University, \\ Zhengzhou, Henan 450052, P.R. China
}

Received May 20, 2014; Accepted February 17, 2015

DOI: $10.3892 / \mathrm{ol} .2015 .3151$

\begin{abstract}
Dipeptidyl peptidase IV (DPPIV; also known as cluster of differentiation 26) and the surface-expressed protease, seprase [also known as fibroblast activation protein alpha (FAP $\alpha$ )], are able to degrade the extracellular matrix; therefore, they are involved in malignant cell invasion and metastasis. However, the prognostic implications of their overexpression in carcinomas remain controversial. The aim of the present study was to investigate the expression and potential prognostic effects of DPPIV and seprase in cases of ovarian carcinoma. Immunohistochemical analysis (IHC) was performed to assess the protein expression of DPPIV and seprase/FAP $\alpha$ in 199 patients (malignant epithelial ovarian cancer, 128; borderline ovarian tumors, 41; and benign ovarian tumors, 30). In addition, in situ hybridization was used to detect the mRNA expression levels of DPPIV and seprase in 86 malignant epithelial ovarian cancer samples. IHC revealed positive staining for seprase and DPPIV proteins in $110 / 128(85.94 \%)$ and $106 / 128(82.81 \%)$ patients with ovarian cancer, respectively. Seprase and DPPIV protein expression was associated with lymph node metastasis and the International Federation of Gynecology and Obstetrics stage. By contrast, no significant correlation was detected between the proteins and the patient age or histological grade and type of tumor. Immunostaining was stronger in the cancerous tissues compared with the borderline and benign tissues. Increased levels of seprase, but not DPPIV, were significantly associated with a shorter disease-free survival $(\mathrm{P}=0.033)$. Further analysis revealed that $96.5(83 / 86)$ and $97.67 \%(84 / 86)$ of the malignant epithelial ovarian cancer samples stained positively for seprase and DPPIV mRNA, respectively. Therefore, DPPIV and seprase may be involved in the development of ovarian cancer, and that they are potential predictive markers of epithelial ovarian carcinoma.
\end{abstract}

Correspondence to: Dr Mengzhen Zhang, Department of Obstetrics and Gynecology, The First Affiliated Hospital of Zhengzhou University, 1 Jianshe East Road, Zhengzhou, Henan 450052, P.R. China

E-mail: zhangmengzhen1@126.com

Key words: seprase, dipeptidyl peptidase IV, ovarian carcinoma

\section{Introduction}

Ovarian cancer is one of the most commonly diagnosed cancer types of the female genital tract (1). Its incidence is highest in developed countries, although the risk of ovarian cancer may be increased by high parity and the use of oral contraceptives (2). Epithelial ovarian cancer (EOC) accounts for 85-90\% of all ovarian carcinomas (3). EOC is often asymptomatic and, currently, no well-established strategies exist for its early detection. The disease spreads readily by direct exfoliation of cells throughout the peritoneal cavity and often recurs at the surface of the peritoneum. Furthermore, it may spread via the lymphatic and hematogenous routes prior to causing any symptoms (4). The majority of patients with ovarian carcinoma are diagnosed with advanced-stage disease. Although optimal cytoreductive surgery and improved chemotherapy have increased the five-year survival rates, the overall survival gains have been limited due to an inability to eradicate all the cancer cells. Therefore, out of all the gynecological cancer types, ovarian carcinoma remains the leading cause of mortality (5). The precise mechanisms underlying invasion and metastasis remain poorly understood. Therefore, it is important to clarify the mechanism of dissemination in order to increase survival and prevent the metastasis and dissemination of ovarian carcinoma cells.

Degradation of the extracellular matrix is required for tumor growth and metastasis $(6,7)$. It is primarily regulated by a variety of proteinases, which are known to influence cellular activities, migration and invasion $(8,9)$. Dipeptidyl peptidase IV (DPPIV) and seprase/fibroblast activation protein $\alpha$ (FAP $\alpha$ ) are membranous serine-type membrane peptidase (SIMP), type II transmembrane proteins with multiple functions, including dipeptidase activities (10). A number of studies have revealed that DPPIV is expressed in cancer cells and is involved in tumor progression and invasion $(11,12)$. Although ovarian carcinoma is a malignancy of the female genital tract with one of the highest mortality rates, studies concerning seprase and DPPIV expression in ovarian carcinoma tissues are lacking. The practical significance of these genes in patients with ovarian cancer remains largely unknown and, therefore, requires further investigation. The aim of the present study was to investigate the expression of DPPIV [also known as cluster of differentiation (CD)26] and seprase/FAP $\alpha$ in EOC cells at the protein and mRNA levels. In addition, the association between the DPPIV and seprase expression levels and known clinicopathological 
prognosticators, including the tumor International Federation of Gynecology and Obstetrics (FIGO) stage, tumor grade and disease outcome, were assessed in order to determine their clinical value.

\section{Materials and methods}

Ovarian carcinoma cell lines. The commercial ovarian carcinoma cell lines, OVCA-3 and SKOV-3, were provided by the Cell Bank of the Chinese Academy of Sciences (Shanghai, China). Immunohistochemical analysis (IHC) and reverse transcription-polymerase chain reaction (RT-PCR) were used to determine the mRNA and protein expression levels of DPPIV and seprase in the cell lines.

Patients and samples. The present study included 199 patients with epithelial ovarian tumors who underwent surgery at the First Affiliated Hospital of Zhengzhou University (Zhengzhou, China) between 2005 and 2010. A total of 199 formalin-fixed and paraffin-embedded tumor specimens were collected. The study was approved by the Institutional Ethics Review Board of Zhengzhou University (Zhengzhou, China). In addition, 31 fresh ovarian cancer tissue samples were frozen and stored at $-80^{\circ} \mathrm{C}$. The tissue sections were stained with antibodies and hematoxylin and eosin (Sigma-Aldrich, St. Louis, MO, USA) for morphological evaluation. Clinicopathological information was retrieved from the medical records of the patients. Staging was performed according to the FIGO guidelines (FIGO, 2000) (13). The clinical features of the patients are summarized in Table I. This study was approved by the Ethics Committee of The First Affiliated Hospital of Zhengzhou University and was performed according to the Declaration of Helsinki. Written informed consent was obtained from each patient's family.

Characteristics of antibodies used. The rat monoclonal antibodies against DPPIV and seprase were obtained from Abcam (cat. nos. ab119346 and ab53066, respectively; Cambridge, MA, USA). Subsequent to optimizing the antibody dilutions, IHC was performed using a LabVision Autostainer 720 (Thermo Fisher Scientific, Waltham, MA, USA). The deparaffinized sections were microwaved in $10 \mathrm{mM}$ citrate buffer ( $\mathrm{pH}$ 6.0) in order to expose the epitopes. Following a 5 -min incubation with $0.03 \%$ hydrogen peroxide to block endogenous peroxidase activity, the slides were washed with Tris-buffered saline (TBS) and incubated with the primary anti-DPPIV and anti-seprase rat monoclonal antibodies (dilution, 1:75) at $4^{\circ} \mathrm{C}$ overnight. Next, the slides were incubated with the rabbit anti-rat immunoglobulin $\mathrm{G}$ (IgG; dilution, 1:200; cat. no. ab6728) secondary antibody (Abcam) for $30 \mathrm{~min}$. The slides were then incubated with a peroxidase-labeled polymer and conjugated to a goat anti-rabbit antibody (dilution 1:200; cat. no. ab150081; Abcam) for $30 \mathrm{~min}$. Next, the slides were stained with 3,3'-diaminobenzidine tetrahydrochloride (Sigma-Aldrich) for $10 \mathrm{~min}$, counterstained with hematoxylin, dehydrated and then mounted in Diatex mounting medium (Diatex S.A., Lyon, France).

The ovarian carcinoma DPPIV-positive and seprase-positive cell lines, OVCAR-3 and SKOV-3, were used as positive controls in the present study. The negative controls consisted of the replacement of the primary antibody with IgG at the same concentration and from the same source. All the controls provided satisfactory results.

The IHC results were evaluated according to the pattern, intensity and extent of staining as follows: i) Staining pattern, demonstrating the presence of membranous, cytoplasmic or nuclear staining; ii) extent of staining, represented with the following scale: 0 , no staining; 1 , staining of $<10 \%$ of cancer cells; 2, staining of $11-50 \%$ of cancer cells; and 3 , staining of $>50 \%$ of cancer cells. Overall, at least 300 cells were analyzed; and iii) staining intensity, represented with the following scale: 0 , negative; 1 , weak; 2 , moderate; and 3 , strong. After setting the aforementioned scoring criteria of the tumor cells, the IHC results were scored according to the intensity and extent of staining by two independent senior pathologists who were blinded to the clinicopathological data. The discordant scores were re-evaluated, and the consensus score was used for further analysis. Based on the intensity and extent of staining, the IHC results were scored between 0 and 3 as follows: 0 , negative; 1 , weak; 2 , moderate; and 3 , strong.

In situ mRNA hybridization. Specific primers (listed in Table II), including the T7 RNA polymerase promoter sequence at the $5^{\prime}$ end, were designed using Primer Premier version 5.0 and synthesized. PA15His (full length seprase cDNA) and pcD26His (DPPIV) were obtained from Dr Wen-Tian Chen (State University of New York at Stony Brook, Stony Brook, NY, USA) and used to prepare the DNA templates. The PCR-produced DNA templates and the primers, which included the T7 RNA polymerase promoter site at their 5' end, were used for in vitro transcription according to the In vitro Transcription T7 Kit (Ambion Life Technologies; Austin, TX, USA) manufacturer's instruction. Subsequently, anti-sense and sense fluorescein isothiocyanate (FITC)-cRNA probes (Ambion Life Technologies) were synthesized against DPPIV and seprase.

Sections measuring $5 \mu \mathrm{m}$ were cut, mounted onto poly-L-lysine coated slides and air-dried. Following dewaxing and rehydration, the sections were treated with $0.3 \%$ Triton X-100 (Sigma-Aldrich) for $15 \mathrm{~min}$ at room temperature, followed by a 20 -min treatment with $500 \mu \mathrm{g} / \mu \mathrm{l}$ proteinase $\mathrm{K}$ (Takara, Dalian, China) at $37^{\circ} \mathrm{C}$. Next, the samples were incubated with 0.1 M glycine in phosphate-buffered saline (PBS) for $5 \mathrm{~min}$ and then $4 \%$ paraformaldehyde in $0.1 \mathrm{M}$ PBS for an additional $5 \mathrm{~min}$ at room temperature for fixation.

Prehybridization was performed in $20 \mu \mathrm{l}$ of the prehybridization solution (Ambion Life Technologies) at $37^{\circ} \mathrm{C}$ for $1 \mathrm{~h}$, following a 10 -min treatment with $0.25 \%$ acetic anhydride in $0.1 \mathrm{M}$ triethanolamine- $\mathrm{HCl}$ buffer $(\mathrm{pH} 8.0)$ at room temperature. The tissues were incubated overnight in $10 \mu 1$ hybridization solution along with FITC-cRNA-probes coated with Sigmacote (Sigma-Aldrich) at $37^{\circ} \mathrm{C}$. The hybridization tissues were incubated with normal rabbit serum (dilution, 1:25; cat. no. ab7487; Abcam), anti-FITC antibodies (dilution, 1:300; cat. no. ab19224; Abcam), rabbit anti-mouse IgG (dilution, 1:50; cat. no. ab6728; Abcam) and alkaline phosphatase-anti-alkaline phosphatase (dilution, 1:50; cat. no. ab95462; Abcam) antibodies. The sections were stained with a NBT/BCIP mixture (Sigma-Aldrich) and then 
Table I. Clinicopathological features and expression levels of seprase and DPPIV proteins in patients with epithelial ovarian cancer.

\begin{tabular}{|c|c|c|c|c|c|c|c|}
\hline \multirow[b]{2}{*}{ Parameters } & \multirow{2}{*}{$\begin{array}{c}\text { Case number } \\
(\mathrm{n}=128), \mathrm{n}\end{array}$} & \multicolumn{3}{|c|}{ DPPIV proteins } & \multicolumn{3}{|c|}{ Seprase proteins } \\
\hline & & Positive (\%) & $\chi^{2}$-value & P-value & Positive (\%) & $\chi^{2}$-value & P-value \\
\hline FIGO stage & & & 9.311 & $0.01^{\mathrm{a}}$ & & 6.865 & $0.032^{\mathrm{a}}$ \\
\hline I & 31 & $21(67.7)$ & & & $21(72.4)$ & & \\
\hline II & 40 & $32(80.0)$ & & & $35(85.4)$ & & \\
\hline III & 57 & $53(90.0)$ & & & $54(93.1)$ & & \\
\hline Histology & & & 2.326 & 0.313 & & 3.188 & 0.203 \\
\hline Serous & 78 & $67(85.9)$ & & & $69(88.5)$ & & \\
\hline Mucous & 41 & $33(80.5)$ & & & $35(85.4)$ & & \\
\hline Others & 9 & $6(66.7)$ & & & $6(66.7)$ & & \\
\hline Grade & & & 2.889 & 0.236 & & 0.74 & 0.691 \\
\hline G1 & 27 & $20(74.1)$ & & & $22(81.5)$ & & \\
\hline G2 & 41 & $33(80.5)$ & & & $35(85.4)$ & & \\
\hline G3 & 60 & $53(88.3)$ & & & $53(88.3)$ & & \\
\hline Age/year & & & 0.003 & 0.959 & & 0.08 & 0.778 \\
\hline$\geq 60$ & 75 & $62(82.7)$ & & & $65(86.7)$ & & \\
\hline$\leq 60$ & 53 & $44(83.0)$ & & & $45(84.9)$ & & \\
\hline LN & & & 4.23 & $0.04^{\mathrm{a}}$ & & 6.223 & $0.013^{\mathrm{a}}$ \\
\hline Positive & 48 & $44(91.7)$ & & & $46(95.8)$ & & \\
\hline Negative & 80 & $62(77.5)$ & & & $64(80.0)$ & & \\
\hline
\end{tabular}

${ }^{a} \mathrm{P}<0.05$ indicates a statistically significant difference. DDPIV, dipeptidyl peptidase IV; FIGO, International Federation of Gynecology and Obstetrics; LN, lymph node metastasis.

counterstained with fast red (Sigma-Aldrich). A positive reaction in this assay was indicated by blue staining. The aforementioned positive controls and sense probes were used as positive and negative controls for each hybridization. The scoring criteria were similar to those used for the IHC.

Microselection. A microselection method $(14,15)$ was used to avoid normal elements and areas with prominent infiltration of lymphoid cells. In total, $5-\mu \mathrm{m}$ frozen sections were cut and stained with hematoxylin and eosin. Next, a region of tumor cells in the sections was selected using the Nikon Eclipse 50i microscope (Nikon Inc; Melville, NY, USA). The corresponding area on the frozen blocks was then marked and oriented. Next, the block was trimmed in order to calculate the tumor cell area. The trimmed frozen block was re-embedded with O.C.T. $^{\text {Tм }}$ compound (Tissue-Tek, Torrance, CA, USA), and a new $5-\mu \mathrm{m}$ frozen section was created from the re-embedded block to ensure that the cancer cells occupied $>80 \%$ of the selected area. Next, $20-\mu \mathrm{m}$ frozen sections were cut and transferred into cooled Eppendorf tubes. Subsequent to section collection, an additional $5-\mu \mathrm{m}$ frozen section was cut and stained with hematoxylin and eosin for morphological analysis. If normal elements were apparent, the sections were not used, and a different region was selected for microselection.

$R T-P C R$. Total RNA was extracted from microselected carcinoma tissues of patients with ovarian carcinoma using the
RNeasy Mini kit (Qiagen, Valencia, CA, USA), according to the manufacturer's instructions.

The primer pairs for seprase and DPPIV mRNA (previously mentioned) were used in the present study without the T7 RNA polymerase promoter sequence at the $5^{\prime}$ end. The PCR reactions were performed in a $25-\mu 1$ reaction mixture using the Qiagen OneStep RT-PCR kit (Qiagen), according to the manufacturer's instructions (Table II). GAPDH primers were used as an internal control to generate an amplified $105 \mathrm{bp}$ PCR fragment. The program included steps for reverse transcription and PCR, starting with reverse transcription of RNA at $50^{\circ} \mathrm{C}$ for $30 \mathrm{~min}$. PCR amplification consisted of an initial heating step at $95^{\circ} \mathrm{C}$ for $15 \mathrm{~min}$ in order to activate the HotStar Taq DNA polymerase, deactivate the reverse transcriptases and denature the cDNA. The cDNA was then subjected to 35 cycles of denaturation at $94^{\circ} \mathrm{C}$ for $40 \mathrm{sec}, 56^{\circ} \mathrm{C}$ for $40 \mathrm{sec}$ and $72^{\circ} \mathrm{C}$ for $1 \mathrm{~min}$ for primer extension. The PCR products were visualized by electrophoresis on a $7.5 \%$ polyacrylamide gel. The GAPDH primers were used as loading controls for each sample. For the negative control, water was used instead of the template RNA. The OVCAR-3 and SKOV-3 samples were used as the positive controls as they are known to be seprase- and DPPIIV-positive. The positive and negative controls produced satisfactory results in all the series. The images were then semi-quantitatively analyzed by comparing the intensity of the amplified seprase and DPPIV bands with the control GAPDH band. Subsequently, the ratio of the intensities of the DPPIV, seprase and GAPDH bands 
Table II. Polymerase chain reaction primers containing T7 RNA polymerase promoter sequence at the 5' end.

\begin{tabular}{|c|c|c|c|}
\hline Primers & Primer sequences & $\begin{array}{l}\text { Product } \\
\text { length }\end{array}$ & $\begin{array}{l}\text { Annealing } \\
\text { temperature }\end{array}$ \\
\hline \multicolumn{4}{|l|}{ 1-DPPIV } \\
\hline \multirow[t]{2}{*}{$\begin{array}{l}\text { Antisense } \\
\text { probes }\end{array}$} & $\begin{array}{l}\text { F: (T7) 5'-TAA TAC GAC TCA CTA TAGG-ACT-GAA-CTG-GGC } \\
\quad \text {-CAC-TTA-CC-3' }\end{array}$ & & \\
\hline & R: 5'-GTT-ACG-TAC-CCT-CCA-TAT-GAC-C-3' & $247 \mathrm{bp}$ & $58^{\circ} \mathrm{C}$ \\
\hline \multicolumn{4}{|l|}{ 2-DPPIV } \\
\hline \multirow[t]{2}{*}{ Sense probes } & F: 5'-ACT-GAA-CTG-GGC-CAC-TTA-CC-3' & & \\
\hline & $\begin{array}{l}\text { R: (T7) 5'- TAA TAC GAC TCA CTA TAGG-GTT-ACG-TAC-CCT } \\
\text {-CCA-TAT-GAC-C 3' }\end{array}$ & $247 \mathrm{bp}$ & \\
\hline \multicolumn{4}{|l|}{ 3-Seprase } \\
\hline \multirow[t]{2}{*}{$\begin{array}{l}\text { Anti-sense } \\
\text { probes }\end{array}$} & $\begin{array}{l}\text { F: (T7) 5'-TAA TAC GAC TCA CTA TAGG-GAT-TCT-TCC-TCC } \\
\text {-TCA-ATT-TG-3' }\end{array}$ & & \\
\hline & R: 5'-GTC-ACC-TTG-GAA-AGC-TGT-TC-3' & $190 \mathrm{bp}$ & $58^{\circ} \mathrm{C}$ \\
\hline \multicolumn{4}{|l|}{ 4-Seprase } \\
\hline Sense probes & $\begin{array}{l}\text { F: 5'-GAT-TCT-TCC-TCC-TCA-ATT-TG-3' } \\
\text { R: (T7) 5'-TAA TAC GAC TCA CTA TAGG -GTC-ACC-TTG-GAA } \\
\quad \text {-AGC-TGT-TC-3' }\end{array}$ & $190 \mathrm{bp}$ & \\
\hline
\end{tabular}

F, forward; R, reverse; bp, base pairs.

was recorded and divided into the following three grades: low, + ; moderate, ++ ; and high, +++ .

Western blot analysis. The $20-\mu \mathrm{m}$ frozen sections obtained from the microselected tissues were homogenized in the lysis buffer (Beyotime, Shanghai, China) to preserve the integrity and phosphorylation activity of the proteins. The lysis buffer consisted of $1 \%$ NP40, 10\% glycerol, $20 \mathrm{mM}$ Tris- $\mathrm{HCl}$ (pH 7.5), $137 \mathrm{mM} \mathrm{NaCl}, 100 \mathrm{mM} \mathrm{NaF}, 1 \mathrm{mM}$ sodium vanadate, $1 \mathrm{mM}$ phenylmethylsulfonyl fluoride and $10 \mu \mathrm{g} / \mathrm{ml}$ each of leupeptin, pepstatin and aprotinin. Next, the lysates were sonicated at a power setting of $50 \mathrm{~W}$ for a duration of $5 \mathrm{~s}$, with intervals of $15 \mathrm{~s}$, repeated six times, and clarified by centrifugation $\left(10,000 \times \mathrm{g}, 15 \mathrm{~min}, 4^{\circ} \mathrm{C}\right)$, followed by protein quantification using Bradford analysis.

The samples (20 $\mu \mathrm{g}$ proteins/each lane) were separated by $7.5 \%$ SDS-PAGE and then blotted onto Millipore immobilon-P membranes (EMD Millipore, Billerica, MA, USA). Next, the membranes were blocked for $1 \mathrm{~h}$ at room temperature with 5\% non-fat dry milk in TBS/Tween-20 (TBST). The membranes were incubated overnight at $4^{\circ} \mathrm{C}$ with mAb D8 (dilution, 1:800) and E26 (dilution, 1:800). A mouse monoclonal anti- $\beta$-actin antibody (mouse IgG1 isotype; Sigma-Aldrich) was used as the loading control. The reactions with the anti-rat and anti-mouse $\operatorname{IgG}$ (heavy + light chains) horseradish peroxidase conjugates (Promega Corporation, Madison, WI, USA) diluted with TBST (dilution, 1:5000), in addition to the washing color reaction, were assessed using the ECL Plus Western Blotting Detection Reagent kit (GE Healthcare Life Sciences, Little Chalfont, UK). Subsequently, the blots were exposed in an X-ray film cassette (Dskar Health Care Co., Ltd.; Guangzhou, Guangdong, China) and immediately developed.
The negative controls consisted of antibodies in the absence of lysate, whereas the positive controls consisted of samples from the OVCAR-3 and SKOV-3 cell lines.

The images were semi-quantitatively analyzed by determining the ratio of DPPIV and seprase to actin. The intensity ratio of the DPPIV or seprase bands and the $\beta$-actin band was recorded and divided into three grades, as follows: low, +; moderate, ++ ; and high, +++ .

Statistical analysis. Statistical analyses were performed using the SPSS version 17.0 statistical software (SPSS Inc., Chicago, IL, USA). The statistical significance of the intergroup differences was evaluated using the $\chi^{2}$ test. Survival analyses were performed using the Kaplan-Meier product-limit method, and the differences were evaluated using the log-rank test. The disease-free survival period accounted for the time between surgery and first recurrence, metastasis or mortality. To further investigate the relationship between overall survival and the prognostic factors, a Cox proportional hazard model was applied. All the statistical tests were two-sided. A value of $\mathrm{P}<0.05$ was considered to indicate a statistically significant difference.

\section{Results}

IHC of seprase and DPPIV. IHC identified positive staining for the DPPIV protein in 106 of the 128 patients $(85.94 \%)$ with ovarian cancer. This was significantly higher compared with the results observed for the borderline ovarian tumor (56.09\%) and benign ovarian tumor (16.66\%) patients. In total, 22 (17.19\%), $28(21.88 \%), 40(31.25 \%)$ and $38(29.69 \%)$ of the 128 tissue samples from ovarian cancer patients demonstrated negative, weak, moderate and strong DPPIV protein expression, respectively. Furthermore, 116 of the 128 cases $(85.94 \%)$ demonstrated 
Table III. DPPIV and seprase expression levels in different ovarian tissues.

\begin{tabular}{|c|c|c|c|c|c|c|c|c|c|c|c|}
\hline \multirow[b]{2}{*}{ Groups } & \multirow[b]{2}{*}{$\mathrm{n}$} & \multicolumn{5}{|c|}{ DPPIV } & \multicolumn{5}{|c|}{ Seprase } \\
\hline & & - & + & ++ & +++ & $\%$ & - & + & ++ & +++ & $\%$ \\
\hline Benign tumor & 30 & 25 & 3 & 2 & 0 & 16.66 & 24 & 4 & 2 & 0 & 20.00 \\
\hline Borderline tumor & 41 & 18 & 14 & 7 & 2 & 56.09 & 16 & 12 & 12 & 1 & 60.97 \\
\hline Malignant tumor & 128 & 22 & 28 & 40 & 38 & $82.81^{\mathrm{a}}$ & 18 & 35 & 25 & 50 & $85.93^{\mathrm{a}}-\mathrm{l}$ \\
\hline
\end{tabular}

DPPIV, dipeptidyl peptidase IV. ${ }^{\text {a }}<0.05$, vs. benign tumor.
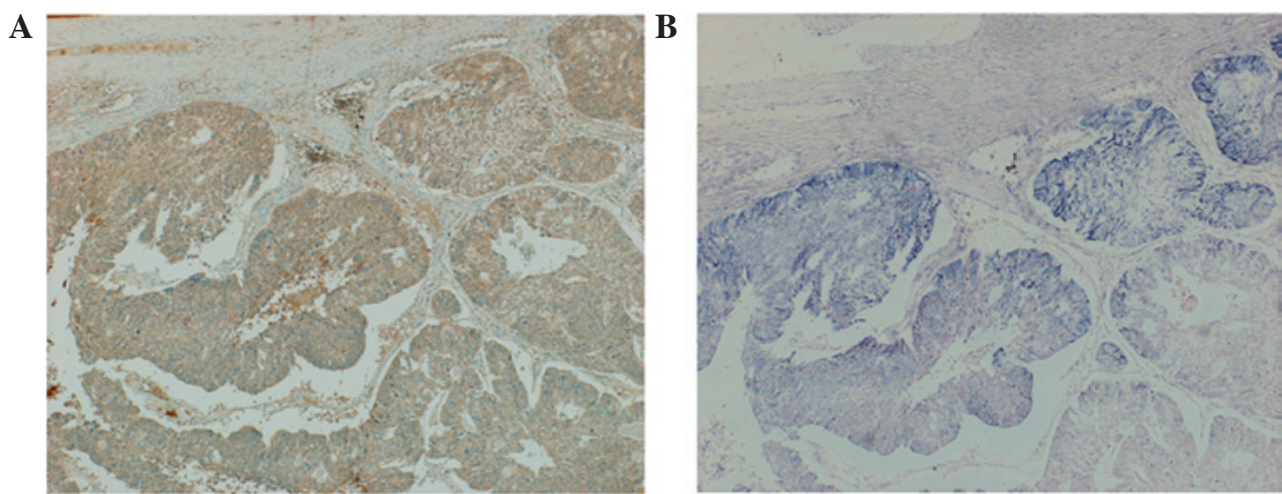

C
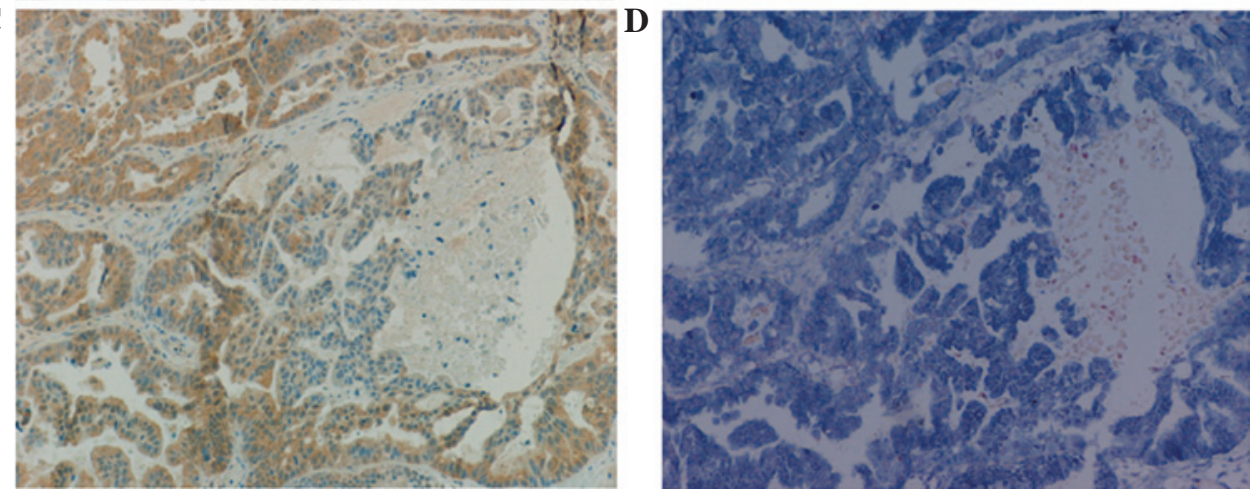

Figure 1. IHC and ISH revealing the expression and location of DPPIV and seprase protein and mRNA levels in ovarian carcinoma cells. Results of DPPIV (A) IHC (magnification, x100) and (B) ISH, (magnification, x100). Results of seprase (C) IHC (magnification, x200) and (D) ISH (magnification, x200) in the cytoplasm. IHC, immunohistochemical analysis; ISH, in situ hybridization; DPPIV, dipeptidyl peptidase IV.

positive staining for seprase, while 18 (14.06\%), 35 (27.34\%), 25 $(19.53 \%)$ and $50(39.06 \%)$ of the 128 patients were negative, weak, moderate or strong for seprase protein expression, respectively.

Although both the cancer and reactive mesothelial cells expressed seprase and DPPIV, the levels were consistently higher in the carcinoma cells. Protein expression was cytoplasmic and/or membranous in the tumor cells, whereas it was exclusively cytoplasmic in the mesothelial cells. Furthermore, DPPIV and seprase were found to often co-localized in the same tumor regions (Fig. 1), and a positive correlation was identified between DPPIV and seprase protein expression $\left(r_{s}=0.504\right.$, $\mathrm{P}=0.001$ ). Higher expression levels of the proteins were identified in the cancer tissues when compared with the levels in borderline ovarian tumors $(56.09 \%)$ or benign ovarian tumors (16.66\%; Table III).

Association between seprase and DPPIV immunoreactivity and clinical pathological features. In order to examine the clinicopathological significance of seprase and DPPIV expression, their association with a number of clinicopathological factors was investigated. The expression levels of the seprase and DPPIV proteins increased with increasing FIGO stage $(\mathrm{P}=0.013$ and $\mathrm{P}=0.023)$. Furthermore, the expression levels of seprase and DPPIV were significantly higher in the EOC patients with lymph node metastasis compared with those without lymph node metastasis. However, this observation did not correlate with the histological grade $(\mathrm{P}>0.05)$. In addition, no significant difference was detected among various age groups and histological types for seprase or DPPIV (Table I).

Seprase and DPPIV mRNA expression levels by in situ hybridization (ISH). ISH using FITC-labeled cRNA probes was used to detect signals in the cancer and stromal cells. In the control experiments, which used sense probes, signals were not detectable in any tissue region. Of the 86 EOC samples, 


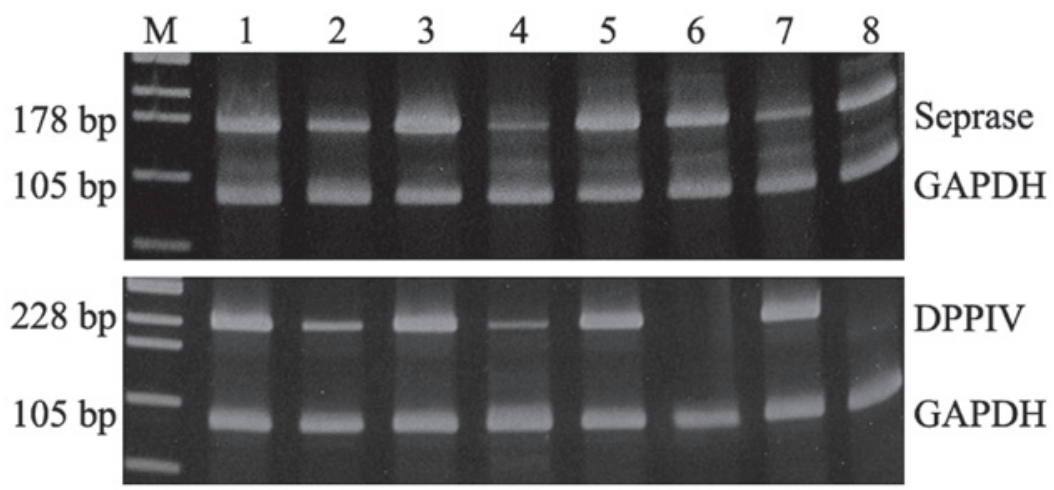

Figure 2. DPPIV and seprase reverse transcription-polymerase chain reaction products in ovarian tumors and carcinoma cell lines. Seprase (top panel): Lane 1, ovarian carcinoma OVCA-3 cell line (+++); lanes 2 and 7, two representative samples for the borderline tumor tissue (+); lanes 3,5 and 6 , three representative samples for the ovarian carcinoma tissue (++ and +++); lane 4, benign ovarian tumor tissue (+); lane 8, ovarian carcinoma SKOV-3 cell line (++). DPPIV (bottom panel): Lane 1, ovarian carcinoma OVCA-3 cell line (+++); lanes 2 and 4, two representative samples for the borderline tumor tissue (++, + ); lanes 3 , 5 and 7, three representative samples for the ovarian carcinoma tissue (+++); and lanes 6 and 8, two representative samples for the benign ovarian tumors (-). DDPIV, dipeptidyl peptidase IV.

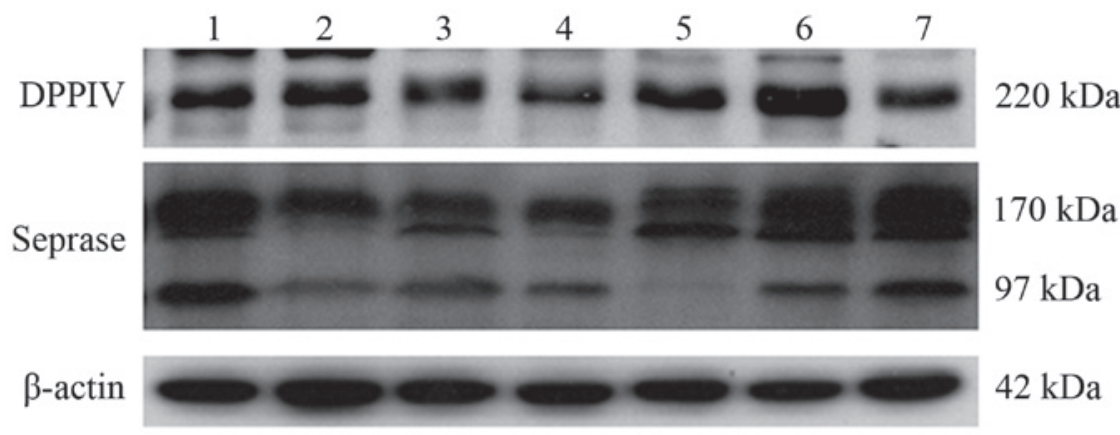

Figure 3. Western blotting results for the ovarian carcinoma samples and cell lines. DPPIV (top panel): Lane 1, ovarian carcinoma OVCAR3 cell line (++); lane 2, ovarian carcinoma SKOV3 cell line $(++)$; lane 3, borderline ovarian tumor tissue $(+)$; lane 4 , benign ovarian tumor tissue $(+)$; lanes 5 and 7 , two representative samples for the ovarian carcinoma tissue (++); lane 6, ovarian carcinoma tissue (+++). Seprase (middle panel): Lane 1, ovarian carcinoma OVCAR3 cell line $(++)$; lane 2, ovarian carcinoma SKOV3 cell line $(++)$; lane 3, borderline ovarian tumor tissue $(++)$; lane 4, benign ovarian tumor tissue $(+)$; lanes 5-7, three representative samples for the ovarian carcinoma tissues $(+++)$. $\beta$-actin (bottom panel). DDPIV, dipeptidyl peptidase IV.

$96.5 \%(83 / 86)$ stained positive for seprase mRNA by ISH. Based on the results of the semi-quantitative RT-PCR, 3 (3.49\%), 21 (24.42\%), 36 (41.86\%) and 26 (30.23\%) of the tumors exhibited negative, weak, moderate or strong seprase mRNA expression, respectively. Seprase mRNA expression was correlated with the corresponding protein as demonstrated by the IHC results $\left(r_{s}=0.48, \mathrm{P}=0.001\right)$. As for DPPIV, $2(2.33 \%), 18(20.93 \%), 40(46.51 \%)$ and $26(30.23 \%)$ of the cases demonstrated negative, weak, moderate or strong mRNA expression, respectively. A significant association was observed between the protein and mRNA expression of DPPIV and seprase $\left(r_{s}=0.66, \mathrm{P}=0.001\right)$. In addition, the mRNA expression profiles were similar to the protein levels.

$R T$-PCR. Variable levels of seprase mRNA were observed among the 31 tumors that were analyzed. Of those, 29 cases demonstrated a degree of DPPIV expression following microselection-assisted RT-PCR (Fig. 2).

Immunoblotting. In total, 25 out of the 31 tumor samples were detected to present the dimeric (170-kDa) and monomeric (97-kDa) forms of seprase. However, following western blot analysis, only one case exhibited the $170-\mathrm{kDa}$ dimeric form alone. A 200-220 kDa DPPIV form was identified in 29 out of the 31 samples (Fig. 3). The DPPIV and seprase protein were detected in the human EOC cell lines, SKOV3 and OVCAR3.

Survival. Univariate analysis of the 128 patients included in this cohort revealed that increased levels of seprase, but not DPPIV, were significantly associated with a decreased probability of disease-free survival ( $\mathrm{P}=0.03$ and $\mathrm{P}=0.52$, respectively; Fig. 4). The survival rates of the patients within each histological subgroup did not differ between the seprase-positive and -negative tumors (data not shown). Therefore, several variables were investigated in order to evaluate whether they had an impact on survival. In the Cox multivariate analysis of the FIGO stage, age groups and histological grade were included, with seprase being an independent risk factor for poor outcome.

\section{Discussion}

DPPIV, seprase/FAP $\alpha$ and other associated prolyl serine peptidases are serine-type integral membrane peptidases (SIMPs) (10). SIMPs exhibit high structural homology, with a cytoplasmic tail that contains six amino acids (a.a.), a 


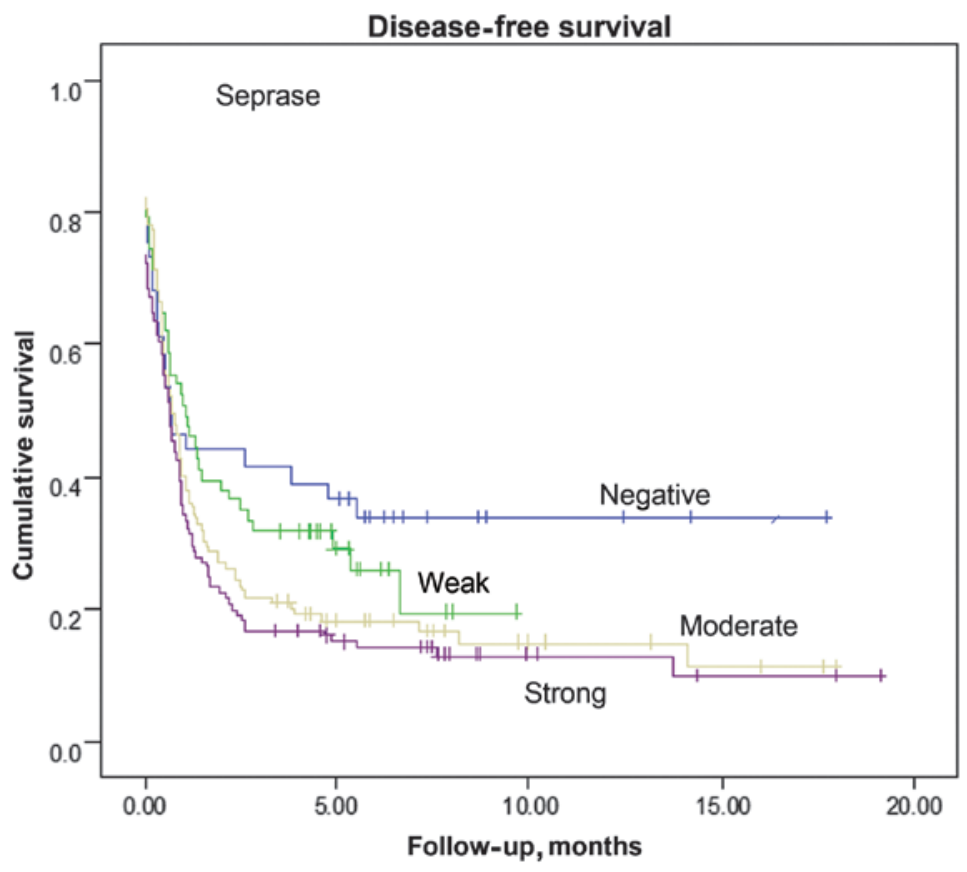

Figure 4. Kaplan-Meier survival curves revealing the negative correlation between increased seprase protein expression and poor disease outcome.

20-a.a. (seprase) or 22-a.a. (DPPIV) transmembrane domain at the $\mathrm{N}$ terminus, an $\mathrm{N}$-glycosylated and cysteine-rich substrate-binding domain and a 200-a.a. region at the C-terminus, which contains the catalytic region consisting of a catalytic serine in a non-classical orientation (16). DPPIV and seprase/FAP $\alpha$ share $68 \%$ of their identity at the catalytic region and the conserved serine protease motif, Gly-Trp-SerTyr-Gly (15). A previous study has revealed that DPPIV and seprase/FAP $\alpha$ cleave prolyl peptide bonds (17). Although it is unclear how they are activated, dimerization is required for prolyl peptidase and gelatinase activities (18). The $\mathrm{N}$-glycosylated and cysteine-rich substrate-binding domains may be important in the recognition of and binding to of substrates. The substrates are proline-containing peptides, including certain growth factors, such as vasoactive peptides, neuropeptides and chemokines (19). Following digestion of the bioactive peptides, DPPIV and seprase/FAP $\alpha$ are able to regulate a number of cellular functions at the cell surface. They function as adhesion molecules and, due to their enzymatic activities, are involved in cell-extracellular matrix interactions and bioactive peptide/cytokine/growth factor metabolism by reducing the activity of chemokines and other peptide mediators (10). DPPIV and seprase exhibit a range of cellular roles, since they are able to form complexes alone or with each other and then interact with other membrane-associated molecules. The localization of protease complexes at cell surface invadopodia is important in the processing of soluble factors, such as neuropeptide Y and certain chemokines (20). Furthermore, this process degrades local extracellular matrix components that are required for cell migration and matrix invasion during tumor invasion, angiogenesis and metastasis (21).

Previous studies have revealed that seprase overexpression was evident in stromal fibroblasts and tumor cells in invasive breast, gastric, colonic and cervical carcinomas; however, it was absent or undetectable in all normal tissue cells, with the exception of cells involved in the early stages of wound healing (22-25). In addition, an in vivo study using a mouse model of human breast cancer, demonstrated that seprase increased microvessel density and promoted rapid tumor growth (26). Another study revealed that the stromal expression of seprase was associated with prolonged survival in patients with invasive ductal carcinoma of the breast (27).

A number of previous studies have reported that DPPIV mRNA and protein are abnormally expressed in a variety of human carcinomas, including prostate, thyroid and colon cancer, as well as endometrial adenocarcinoma; in addition, they were found to be involved in the processes of tumor progression and metastasis (28-32). However, Kajiyama et al (33) identified that nude mice inoculated with DPPIV-transfected SKOV3 cells exhibited significantly less peritoneal dissemination and increased survival times compared with those without transfection (33). This conflicting finding may be the result of short follow-up periods or a lack of samples. Therefore, further studies are required in order to clarify this result.

To the best of our knowledge, the study of DPPIV and seprase in ovarian carcinomas has so far been confined to cell lines (34-36). Furthermore, no large-scale comparative studies concerning seprase expression in specimens of ovarian carcinoma have been conducted. Based on a large series of patients with EOC, to the best of our knowledge, the present study provided the first immunohistochemical evidence that an overexpression of seprase and DPPIV is more often and strongly observed in malignant tissues compared with borderline and benign counterparts (data not shown). In cancerous tissues, positive staining was not only observed in cancer cells, but also in certain stromal spindle cells (fibroblasts) and microvessel endothelial cells adjacent to the cancer cells. However, the immunoreactivity of the cancer cells was consistently stronger compared with that of the stromal spindle and 
microvessel endothelial cells. Immunoreactivity for seprase or DPPIV protein was not evident in the fibroblasts from normal tissues remote from the cancer cells. The pattern of protein expression in the tumor cells was mainly cytoplasmic, although membranous and nuclear expression was occasionally observed. By contrast, the expression was exclusively cytoplasmic in the stromal spindle and microvessel endothelial cells. In certain cases, stronger staining of DPPIV and seprase was present in the malignant cells located at the infiltration front, rather than the central region of the cancer tissue.

In addition, the tumor nests demonstrated diffuse and heterogeneous immunostaining. The DPPIV and seprase proteins often colocalized in the same tumor regions (Fig. 1). Furthermore, a positive correlation was detected between DPPIV and seprase proteins $\left(r_{s}=0.504, \mathrm{P}=0.001\right)$. These observations suggest that ovarian carcinoma cells produce DPPIV and seprase. This supports the hypothesis that DPPIV and seprase are the primary cell-surface enzymes responsible for cellular invasion and are important in ovarian cancer.

Generally, patients with advanced ovarian carcinoma exhibit a poorer survival rate compared with patients at earlier stages. In the present study, the protein expression of seprase and DPPIV was correlated with the FIGO stage and the presence or absence of lymph node metastasis. By contrast, no significant correlation was observed between the two proteins and the patient age, or the histological grade or type of the tumor. In addition, it was revealed that increased seprase protein expression was negatively associated with disease-free survival ( $\mathrm{P}=0.033$ ) (Fig. 4). However, no association was detected between DPPIV protein expression and disease-free survival $(\mathrm{P}=0.521)$.

In the present study, seprase and DPPIV mRNA transcripts were detected in ovarian carcinomas. IHC established that seprase mRNA expression was correlated with its corresponding protein $\left(r_{s}=0.48, \mathrm{P}=0.001\right)$. With respect to DPPIV, a significant correlation was observed between DPPIV mRNA and protein $\left(r_{s}=0.66, \mathrm{P}=0.001\right)$. Although seprase and DPPIV mRNA transcripts were detected, protein immunoreactivity was negative in certain tumors. This discrepancy in the protein expression of seprase and DDPIV in certain tumors may be the result of post-transcriptional regulation by factors such as estrogen.

In conclusion, the results of the present study indicated that seprase and DPPIV are involved in the progression of ovarian cancer. The results assisted the identification of a cohort of patients with EOC that exhibit poorer prognoses. Therefore, DDPIV and seprase may serve as potential prognostic markers for this type of tumor.

\section{Acknowledgements}

This study was supported by grants from the Excellent Youth Foundation of Henan Scientific Committee (no. 104100510007), and the Medical Science and Technique Foundation of Henan Province (no. 201001005).

\section{References}

1. Jemal A, Bray F, Center MM, Ferlay J, Ward E and Forman D: Global cancer statistics. CA Cancer J Clin 61: 69-90, 2011.

2. Aletti GD, Gallenberg MM, Cliby WA, Jatoi A and Hartmann LC: Current management strategies for ovarian cancer. Mayo Clin Proc 82: 751-770, 2007.
3. Morrison J: Advances in the understanding and treatment of ovarian cancer. J Br Menopause Soc 11: 66-71, 2005.

4. Bakrin N, Bereder JM, Decullier E, Classe JM, et al; FROGHI (French Oncologic and Gynecologic HIPEC) Group: Peritoneal carcinomatosis treated with cytoreductive surgery and Hyperthermic Intraperitoneal Chemotherapy (HIPEC) for advanced ovarian carcinoma: a French multicentre retrospective cohort study of 566 patients. Eur J Surg Oncol 39: 1435-1443, 2013

5. Coleman RL, Monk BJ, Sood AK and Herzog TJ: Latest research and treatment of advanced-stage epithelial ovarian cancer. Nat Rev Clin Oncol 10: 211-224, 2013.

6. Werb Z: ECM and cell surface proteolysis: regulating cellular ecology. Cell 91: 439-442, 1997.

7. Talvensaari-Mattila A, Santala M, Soini Y and Turpeenniemi-Hujanen T: Prognostic value of matrix metalloproteinase-2 (MMP-2) expression in endometrial endometrioid adenocarcinoma. Anticancer Res 25: 4101-4105, 2005.

8. Rowe RG and Weiss SJ: Navigating ECM barriers at the invasive front: the cancer cell-stroma interface. Annu Rev Cell Dev Biol 25: 567-595, 2009.

9. Deakin NE and Chaplain MA: Mathematical modeling of cancer invasion: the role of membrane-bound matrix metalloproteinases. Front Oncol 3: 70, 2013.

10. Chen WT, Kelly T and Ghersi G: DPPIV, seprase and related serine peptidases in multiple cellular functions. Curr Top Dev Biol 54: 207-232, 2003

11. Goscinski MA, Suo ZH, Nesland JM, Flørenes VA and Giercksky KE: Dipeptidyl peptidase IV expression in cancer and stromal cells of human esophageal squamous cell carcinomas, adenocarcinomas and squamous cell carcinoma cell lines. APMIS 116: 823-831, 2008.

12. Mentlein R, Hattermann K, Hemion C, Jungbluth AA and Held-Feindt J: Expression and role of the cell surface protease seprase/fibroblast activation protein- $\alpha(\mathrm{FAP}-\alpha)$ in astroglial tumors. Biol Chem 392: 199-207, 2011.

13. Benedet JL, Bender H, Jones H III, Ngan HY and Pecorelli S: FIGO staging classifications and clinical practice guidelines in the management of gynecologic cancers. FIGO Committee on Gynecologic Oncology. Int J Gynaecol Obstet 70: 209-262, 2000 .

14. Wu Q, Suo Z, Risbegr B, Karlsson MG, Villman K and Nesland JM: Expression of EPhb2 And Ephb4 in Berast Caerinoma. Phatol oneol Res 10: 26-33, 2004.

15. Lawrie LC, Curran S, MeLeod HL, Fothergill JE and Murray GI: Applieation of laser capture microdissection And porteomics in colon cancer. Mol Pathol 54: 253-258, 2001.

16. Monsky WL, Lin CY, Aoyama A, Kelly T, Akiyama SK, Mueller SC and Chen WT: A potential marker protease of invasiveness, seprase, is localized on invadopodia of human malignant melanoma cells. Cancer Res 54: 5702-5710, 1994.

17. Chen WT and Kelly T: Seprase complexes in cellular invasiveness. Cancer Metastasis Rev 22: 259-269, 2003.

18. Kotacková L, Baláziová E and Sedo A: Expression pattern of dipeptidyl peptidase IV activity and/or structure homologues in cancer. Folia Biol (Praha) 55: 77-84, 2009.

19. Pro B and Dang NH: CD26/dipeptidyl peptidase IV and its role in cancer. Histol Histopathol 19: 1345-1351, 2004.

20. Mueller SC, Ghersi G, Akiyama SK, Sang QX, Howard L, Pineiro-Sanchez M, et al: A novel protease-docking function of integrin at invadopodia. J Biol Chem 274: 24947-24952, 1999.

21. O'Brien P and O'Connor BF: Seprase: an overview of an important matrix serine protease. Biochim Biophys Acta 1784: $1130-1145,2008$

22. Kelly T, Kechelava S, Rozypal TL, West KW and Korourian S: Seprase, a membrane-bound protease, is overexpressed by invasive ductal carcinoma cells of human breast cancers. Mod Pathol 11: 855-863, 1998.

23. Jin X, Iwasa S, Okada K, Mitsumata M and Ooi A: Expression patterns of seprase, a membrane serine protease, in cervical carcinoma and cervical intraepithelial neoplasm. Anticancer Res 23: 3195-3198, 2003

24. Okada K, Chen WT, Iwasa S, Jin X, Yamane T, Ooi A and Mitsumata M: Seprase, a membrane-type serine protease, has different expression patterns in intestinal- and diffuse-type gastric cancer. Oncology 65: 363-370, 2003

25. Wikberg ML, Edin S, Lundberg IV, Van Guelpen B, Dahlin AM, Rutegård $\mathrm{J}$, et al: High intratumoral expression of fibroblast activation protein (FAP) in colon cancer is associated with poorer patient prognosis. Tumour Biol 34: 1013-1020, 2013. 
26. Huang Y, Wang S and Kelly T: Seprase promotes rapid tumor growth and increased microvessel density in a mouse model of human breast cancer. Cancer Res 64: 2712-2716, 2004.

27. Ariga N, Sato E, Ohuchi N, Nagura H and Ohtani H: Stromal expression of fibroblast activation protein/seprase, a cell membrane serine proteinase and gelatinase, is associated with longer survival in patients with invasive ductal carcinoma of breast. Int J Cancer 95: $67-72,2001$.

28. Wilson MJ, Ruhland AR, Quast BJ, Reddy PK, Ewing SL and Sinha AA: Dipeptidylpeptidase IV activities are elevated in prostate cancers and adjacent benign hyperplastic glands. J Androl 21: 220-226, 2000.

29. Khin EE, Kikkawa F, Ino K, Kajiyama H, Suzuki T, Shibata K, et al Dipeptidyl peptidase IV expression in endometrial endometrioid adenocarcinoma and its inverse correlation with tumor grade. Am J Obstet Gynecol 188: 670-676, 2003.

30. Mizokami Y, Kajiyama H, Shibata K, Ino K, Kikkawa F and Mizutani S: Stromal cell-derived factor-1alpha-induced cell proliferation and its possible regulation by CD26/dipeptidyl peptidase IV in endometrial adenocarcinoma. Int J Cancer 110: 652-659, 2004

31. Abe M, Havre PA, Urasaki Y, Ohnuma K, Morimoto C, Dang LH and Dang NH: Mechanisms of confluence-dependent expression of CD26 in colon cancer cell lines. BMC Cancer 11: 51, 2011.
32. Miyake Y, Aratake Y, Sakaguchi T, Kiyoya K, Kuribayashi T, Marutsuka K and Ohno E: Examination of CD26/DPPIV, p53 and PTEN expression in thyroid follicular adenoma. Diagn Cytopathol 40: 1047-1053, 2012.

33. Kajiyama H, Kikkawa F, Suzuki T, Shibata K, Ino K and Mizutani S: Prolonged survival and decreased invasive activity attributable to dipeptidyl peptidase IV overexpression in ovarian carcinoma. Cancer Res 62: 2753-2757, 2002.

34. Kennedy A, Dong H, Chen D and Chen WT: Elevation of seprase expression and promotion of an invasive phenotype by collagenous matrices in ovarian tumor cells. Int J Cancer 124: 27-35, 2009.

35. Lai D, Ma L and Wang F: Fibroblast activation protein regulates tumor-associated fibroblasts and epithelial ovarian cancer cells. Int J Oncol 41: 541-550, 2012.

36. Yang L, Ma L and Lai D: Over-expression of fibroblast activation protein alpha increases tumor growth in xenografts of ovarian cancer cells. Acta Biochim Biophys Sin (Shanghai) 45: 928-937, 2013 